Global COE Hi-Stat Discussion Paper Series 028

$$
\begin{aligned}
& \text { Research Unit for Statistical } \\
& \text { and Empirical Analysis in Social Sciences (Hi-Stat) }
\end{aligned}
$$

Sequential Internet Auctions with Different Ending Rules

Toshihiro Tsuchihashi

February 2009 


\title{
Sequential Internet Auctions with Different Ending Rules
}

\author{
Toshihiro Tsuchihashi*†
}

January 2009

\begin{abstract}
Two ending rules, a soft close and a hard close, exist in Internet auctions. In hard close auctions, each auction ends with a fixed deadline determined by a seller. In soft close auctions, the end time automatically extends if at least one bid is submitted in the last few minutes, so each buyer has an opportunity to reply to other buyers' bids. The reserve prices set by the seller in hard close auctions are higher than the reserve prices in soft close auctions. The result is consistent with data of DS Lite auctions in Yahoo! Japan.
\end{abstract}

\section{Introduction}

Since Onsale and eBay started Internet auctions in 1995, the number of items and the amount of money in transactions have been growing at a high rate. As Internet auctions are widely used to sell a variety of items, many research papers study Internet auctions theoretically or empirically. Lucking-Reiley (2000) provides a good survey of Internet auctions, and he considers public reserve prices, secret reserve prices, time durations and buy prices in detail. Internet auctions have inherent features as compared with traditional auctions like Sotheby's and Christie's. One of the features is that people can hold the auction whenever they want to sell items, and they can quickly re-auction their items with few costs whenever the item remains unsold. In this sense, we can consider Internet auctions as sequential auctions. There are some works which analyze sequential auctions where a seller has a single item. ${ }^{1}$ McAfee and Vincent

\footnotetext{
${ }^{*}$ Graduate School of Economics, Hitotsubashi University; 2-1, Naka, Kunitachi, Tokyo, Japan 186-8601; E-mail:tsucchi1978@yahoo.co.jp

${ }^{\dagger}$ I am very grateful to members in Hitotsubashi Game Theory Workshop and 14th DC conference for helpful comments and suggestions. I am truly indebted to my superviser Akira Okada for his invaluable guidance. Any remaining errors are of course my own responsibility.

${ }^{1}$ There are also many researches which analyze sequential auctions where a seller has many identical items and she sequentially sells one unit of the items in each auction. In this setting, each buyer has single-unit demand, and he exits from the auction once he gets the item. In this context, buyers' bidding strategies and winning
} 
(1997) analyze both sequential auctions in which the stage game is a sealed-bid second-price or first-price auction in the independent private value (IPV) context. ${ }^{2}$ In their model, a seller posts a high reserve price in the first period and decreases reserve prices in consequent periods, so that each buyer is faced with a chance between submitting in the current period and waiting for lower prices in the future. ${ }^{3}$ In the other study, Grant et al. (2006) discuss repeated auctions in the IPV context in which a seller chooses both a reserve price and a duration for each auction. In their model, a seller cannot change a reserve price once she chooses it. They consider an English auction as a stage game in which bidders appear according to a random (Poison) arrival process.

The other feature of Internet auctions is that there are different rules for ending auctions. Two famous rules exist for ending auctions. The first rule is a hard close which ends an auction with a fixed end time determined by a seller. A hard close is adapted by eBay and other auction sites. Another rule is a soft close which automatically extends an end time if at least one bid is submitted in the last few minutes (ten minutes in Amazon and five minutes in Yahoo! Japan), and the auction ends if the last minutes have passed without any bids. A seller can or cannot choose the rules in Internet auctions. 45 Since ending rules change the structure of auctions, a seller may post different reserve prices depending on ending rules. Since McAfee and Vincent (1997) provide a model of Internet auctions with a hard close, and they characterize a perfect Bayesian equilibrium of hard close auctions. In this paper, we construct a model of Internet auctions with a soft close by developing McAfee and Vincent's (1997) model and compare reserve prices. We obtain the two results. First, we show that the seller posts a higher reserve price in hard close auctions than in soft close auctions. The result is consistent with the auctions data of DS Lite in Yahoo! Japan. Second, while a difference between reserve prices in different auctions exists, a timing of the item to be sold is the same independent of ending rules. There

prices are analyzed but the reserve prices are not. The winning prices are theoretically to drift upward (Weber, 1983; Milgrom and Weber, 2000), but they have a tendency to drift downward in the real auctions (Ashenfelter, 1989).

${ }^{2}$ To our knowledge, we do not find any papers which analyze sequential auctions in the common value context.

${ }^{3}$ In a bargaining under one-sided or two-sided uncertainty, the similar results are obtained. See Samuelson (1984), Fudenberg et al (1985) and Gul et al. (1986).

${ }^{4} \mathrm{~A}$ seller can choose an ending rule in Yahoo! Japan auction. Other Internet auction sites, eBay and Amazon, do not allow sellers to choose rules for ending auctions.

${ }^{5}$ While Yahoo! in the U.S also have provided the similar auction system to Yahoo! Japan's, Yahoo! finished the auction services in September 2007. 
are few papers which analyze ending rules. Roth and Ockenfels (2002) and Ockenfels and Roth (2006) examine sniping by comparing English auctions with different ending rules. ${ }^{6}$ Ockenfels and Roth (2006) develop a one-shot English auction model by introducing a probability of a bid to be failed in the last minute, and show that sniping occurs in equilibrium in hard close auctions such as eBay. Roth and Ockenfels (2002) compare bidding in eBay with that in Amazon and find that there is a great deal of sniping on eBay, and considerably less on Amazon.

The reason why we adopt a sequential auction model instead of a one-shot game is that the unsold item can quickly be re-auctioned with no/few costs in Internet auctions. Furthermore, we can see that ending rules do not affect outcomes in one shot case. Though a stage game of Internet auctions is a kind of English (ascending) auction, it can be considered as a sealed-bidsecond-price auction due to a proxy bid system in the IPV context. ${ }^{7}$ We analyze a sequential auction with a soft close in the IPV context developing McAfee and Vincent's (1997) model, and discuss how the two rules change reserve prices. We do not consider a secret reserve price, buy price and a positive minimum increment. ${ }^{8}$ This paper is organized as follows. Section 2 presents the model. Section 3 considers one shot case and a sequential case. Section 4 analyzes stationary linear equilibria. Section 5 compares ending rules and section 6 concludes. All proofs are given in the Appendix.

\section{The Model}

We consider a sequential soft close auction in the IPV context in which each period is a sealedbid-second-price auction with potentially two stages. A seller has a single item to which she evaluates $v_{0}=0$. She chooses a reserve price $r_{t}$ in each period $t$. There are $n$ potential buyers.

\footnotetext{
${ }^{6}$ Sniping is an inherent phenomenon which many buyers submit bids in the very last few minutes even though the item is been auctioning during a few days.

${ }^{7}$ In Internet auctions, the current price is not the highest bid but the second highest bid plus a minimum increment (called "proxy bid system"). Due to a proxy bid system, each bidder suffices to submit his reserve price early in the auction and wait for the auction end.

${ }^{8}$ Internet auctions equip with some useful systems which can support auction transactions such as a proxy bid, a rating system and a watch list. Furthermore, sellers and buyers have many options. Lucking-Reiley (2000) discusses these options, and many research papers study them theoretically or empirically. Choosing a rule for ending auctions may be one of sellers' options. Minimum increments can affect a buyer's strategy. Submitting a bid equal to a seller's true valuation is not a weakly dominant strategy in IPV English auctions which equip with minimum increments. See Roth and Ockenfels (2002).
} 
Buyer $i$ 's valuation of the item $v_{i}$, which is the buyer $i$ 's private information, is independently and identically drawn from $[0,1]$ according to a distribution function $F$. The density function is given by $f=F^{\prime}>0$ (full support). The seller posts a reserve price $r_{t}$ at the beginning of period $t$ (first stage). The buyer with the highest bid over the reserve price $r$ wins the auction, and pays a price $p$ which is higher of the second highest bid and the reserve price. If a winner's value is $v_{i}$ and the item is sold for a price $p$, the seller's payoff is $p$ and the winner's payoff is $v_{i}-p$. In what follows, we refer buyer $v$ as a buyer with reservation value $v$. We assume that no one can submit a bid below the reserve price. If at least one bid is submitted in the first stage, the second stage starts in the current period $t$, where the buyers simultaneously submit bids (second stage). Notice that no one can submit a bid below the second highest bid in the first stage. On the other hand, if no bid is submitted in period $t$, no buyer can obtain the item and the item is re-auctioned without any costs in period $t+1 .{ }^{9} 10$ Both seller's and buyers' payoffs are discounted by a common discounting factor $\delta \in(0,1)$. No buyer can submit a bid after the second stage is shutdown, thus the auction ends with probability 1 in the current period if the second stage starts. ${ }^{11}$

Strategy A history at the beginning (of the first stage) of period $t$ is a sequence of reserve prices and it is denoted by $h_{t} \in H_{t}=\left\{h_{t} \mid h_{t}=\left(r_{1}, \cdots, r_{t}\right)\right\}$, where $h_{0}=\{\emptyset\}$. A (pure) strategy for each buyer is a pair $b=\left(b^{1}, b^{2}\right)$. In period $t$, the buyer decides whether to submit a bid or not in the first stage given his valuation $v$ and a history $h_{t}$ according to $b_{t}^{1}$, which is a function from $[0,1] \times H_{t}$ to $\mathbb{R}_{+} \cup\{\emptyset\}$, where $\{\emptyset\}$ means no bid. Similarly, the buyer decides whether to submit a bid or not in the second stage of period $t$ given his valuation $v$, a history $h_{t}$ and the reserve price $r_{t}$ according to $b_{t}^{2}$, which is a function from $[0,1] \times H_{t} \times \mathbb{R}_{+}$to $\mathbb{R}_{+} \cup\{\emptyset\}$, where $\{\emptyset\}$ means no bid. ${ }^{12}$ We assume $b_{t}^{2}\left(v, h_{t}, r_{t}\right) \geq b_{t}^{1}\left(v, h_{t}\right)$ for all $t$. A (pure) strategy $s$ for

\footnotetext{
${ }^{9}$ Notice that only the final period has two stages and the other periods have only one stage.

${ }^{10}$ Ockenfels and Roth (2006) consider a one-shot auction with two stages. In their model, an auction is held during a period $[0,1]$. The first stage is $[0,1)$, where any buyers can reply to other bids. The second stage is $\{1\}$, where any buyers cannot reply to other bids.

${ }^{11}$ This assumption loses nothing about generality. We can add the third stage in any periods. If some buyers submit bids in the second stage, the ending time automatically extends, and the third stage will be given to all buyers. However, since nothing is discounted within a period and the auction surely ends at the current periods, the added third stage cannot affect the outcome.

${ }^{12}$ McAfee and Vincent (1997) set up a model where no-bid-option is not allowed for the buyer. In their model, however, the buyer can submit a bid below a reserve price, which is not accepted by the seller, thus the bid
} 
the seller is setting a reserve price $r_{t}$ in period $t$ given history $h_{t}$, and it is formally described as a function from $H_{t-1}$ to $\mathbb{R}_{+}$.

We analyze a symmetric perfect Bayesian equilibrium (PBE) of the game. A PBE of the game is represented by a profile $\left(s^{*}, b^{*}, \mu^{*}\right)$ of strategies and the seller's belief. The seller and buyers maximize their conditional expected payoff. Furthermore, we assume that buyers use the weakly dominant strategies if they exist. The seller's belief $\mu^{*}$ is her probability assessment of the highest buyer's valuation, given a history, and it is formally a function from $H_{t-1}$ to the set of distributions over the highest buyer's valuation $[0,1]$. Precisely, the seller's belief is the probability assessment of all buyers' valuations $\Delta\left([0,1]^{n}\right)$. However, the seller is typically interested in the highest order statistics due to the three assumptions: (i) Buyers' valuations are independently distributed; (ii) Buyers are identical; and (iii) Buyers use a symmetric strategy. Precisely, we must consider buyers' beliefs about other buyers' valuations since each buyer has a chance to update his belief after proceeding a period. However, since we assume that buyers submit weakly dominant bids if they exist, and since buyers actually have weakly dominant bids as we will show, thus we omit the buyers' beliefs. We define a weakly dominant bid in Definition 1 below.

\section{Analysis}

First we consider one period case. In this case, a hard close auction is identical with a standard one-shot sealed-bid-second-price auction. In the auction, each buyer $v$ has a weakly dominant strategy in which he submits his true value $v$. If the seller sets a reserve price $R$, her expected payoff is given by:

$$
\Pi_{0}(R)=n\left[R(1-F(R)) G(R)+\int_{R}^{1} v(1-F(v)) g(v) d v\right],
$$

where $G(x)=(F(x))^{n-1}$. By differentiating $\Pi_{0}$ with respect to $R$, we obtain:

$$
R=\frac{1-F(R)}{f(R)} \text {. }
$$

If $F$ is the uniform distribution on $[0,1]$, then $R=1 / 2$.

bellow a reserve price can be considered as no-bid. Furthermore, they call a bid above reserve price a serious bid. 
A soft close auction can be considered as a sealed-bid-second-price auction with two stages. We can analyze it by backward induction. In the second stage, each buyer $v$ has a weakly dominant strategy in which he submits his true value $v$. In the first stage, if buyer $v$ submits a bid $b$, the bid should be equal to or lower than $v$. Otherwise, he might not be able to submit $v$ in the second stage. However, submitting nothing is strictly dominated by the above bid, since the auction ends if the other buyers do not submit any bids. From the view of a seller, this auction can be considered as the standard one-shot sealed-bid-second-price auction. Therefore, the seller chooses the same reserve price as above.

Second, we consider a sequential case of soft close auctions by developing McAfee and Vincent's (1997) sequential second-price auctions. When we consider a buyer's strategy, we must specify both a timing of submitting and a price against to any reserve prices in both the first and the second stage. However, the next two lemmas can simplify an analysis for a buyer's strategy. Before stating lemmas, we define a weakly dominant bid as below.

Definition 1 Fix a history $h_{t}$ and a reserve price $r_{t}$ in period $t$. For a buyer with a reservation value $v$, a pair of $b_{t}^{1}\left(v, h_{t}\right) \neq \emptyset$ in the first stage and $b_{t}^{2}\left(v, h_{t}, r_{t}\right) \neq \emptyset$ is a weakly dominant bid if:

$$
V_{t}\left(\left(b_{t}^{1}, b_{t}^{2}\right), b_{t}^{-} ; v\right) \geq V_{t}\left(\left(\tilde{b}_{t}^{1}, \tilde{b}_{t}^{2}\right), b_{t}^{-} ; v\right)
$$

for all $\left(\tilde{b}_{t}^{1}, \tilde{b}_{t}^{2}\right)$ and $b_{t}^{-}$where $b_{t}^{-}$denotes the other buyers' bids.

The term $V_{t}\left(\left(b_{t}^{1}, b_{t}^{2}\right), b_{t}^{-} ; v\right)$ represents a buyer's expected payoff when his reservation value is $v$, he submits bid $\left(b_{t}^{1}, b_{t}^{2}\right)$ and the other buyers' bids are $b_{t}^{-}$.

Lemma 1 In any PBE, if buyer $v$ submits a bid, submitting his true value $v$ or lower in the first stage $\left(b_{t}^{1}\left(v, h_{t}\right) \leq v\right)$ and submitting his true value $v$ in the second stage $\left(b_{t}^{s}\left(v, h_{t}, r_{t}\right)=v\right)$ is a weakly dominant bid for all $v, h_{t}, r_{t}$ and $t$.

The proof follows a standard proof which submitting a true value is a weakly dominant strategy in sealed-bid-second-price auctions. Similar to standard sealed-bid-second-price auctions, there exist many asymmetric PBE where one bidder submits a very high bid and the 
others' bids are low. In what follows, we restrict attention to a PBE where each buyer submits a weakly dominant bid, where it satisfies $b_{t}^{1}\left(v, h_{t}\right)=v$ and $b_{t}^{2}\left(v, h_{t}, r_{t}\right)=v$, hence, a PBE is symmetry. 13

Thanks to Lemma 1, we can focus only on timing when each buyer should submit a bid in an optimal strategy. Therefore, we can consider a buyer's strategy as $b_{t}^{1}\left(v, h_{t}\right) \in\{v, \emptyset\}$ and $b_{t}^{2}\left(v, h_{t}, r_{t}\right) \in\{v, \emptyset\}$ for all $v, h_{t}, r_{t}$ and $t$.

Lemma 2 In any PBE, following any history $h_{t}$, if buyer $v$ submits $v$ in the first stage, then any buyer $v^{\prime}>v$ submits $v^{\prime}$ in the first stage.

The proof is similar to proofs in Sobel and Takahashi (1983), Cramton (1984) and McAfee and Vincent (1997). By Lemma 1 and Lemma 2, in any PBE, buyer $v$ should use a cut-off strategy in which he submits $v$ in the first stage if and only if $r_{t}$ is equal to or lower than his cut-off value, and submits $v$ in the second stage if the second stage starts. In $j$-th stage of period $t$, buyer $v$ decides a cat-off value given his reservation value $v$ and history $h_{t}$ according to $\beta_{t}^{j}$, which is a function from $[0,1] \times H_{t}$ to $\mathbb{R}_{+}$. By Lemma $2, \beta_{t}^{j}\left(v^{\prime}, h_{t}\right)>\beta_{t}^{j}\left(v, h_{t}\right)$ for all $v^{\prime}>v$, for all $t$ and $j=1,2$. Notice that since the auction surely ends if the second stage starts, buyer $v$ should submit his true value $v$, so $\beta_{t}^{2}\left(v, h_{t}\right)=v$ for all $t$ and $h_{t}$. In what follows, it is sufficient to consider only the cut-off values in the first stage, so we write $\beta_{t}\left(v, h_{t}\right)$ instead of $\beta_{t}^{1}\left(v, h_{t}\right)$. Therefore, we can replace a buyer $i$ 's strategy to

$$
b_{t}^{1}\left(v, h_{t}\right)=\left\{\begin{array}{l}
v \text { if } r_{t} \leq \beta_{t}\left(v, h_{t}\right) \\
\emptyset \text { otherwise }
\end{array}\right.
$$

Lemma 3 Let $\beta_{t}$ be an equilibrium buyer's strategy. There exists a unique value $v_{t}$ such that the support of $\mu\left(h_{t-1}\right)$ is given by $\left[0, v_{t}\right]$, and $r_{t}=\beta_{t-1}\left(v_{t}, h_{t-1}\right)$ given $r_{t}$ for all $h_{t-1}$ and $t>1$ and $v_{1}=1$.

\footnotetext{
${ }^{13}$ Even though $b_{t}^{1}\left(v, h_{t}\right)<v$ and $b_{t}^{2}\left(v, h_{t}, r_{t}\right)=v$ is also a weakly dominant bid, but the outcome is the same as the outcome induced by $b_{t}^{1}\left(v, h_{t}\right)=v$ and $b_{t}^{2}\left(v, h_{t}, r_{t}\right)=v$. Thus, for simplicity, we restrict attention to the weakly dominant bid $b_{t}^{1}\left(v, h_{t}\right)=v$ and $b_{t}^{2}\left(v, h_{t}, r_{t}\right)=v$.
} 
Lemma 3 determines a way to update the seller's on-path belief. We assume that $v_{t} \in[0,1]$ for all $h_{t}$ and $t$, and the seller's off-path belief is also given by the way in Lemma 3. Furthermore, we can consider as if the seller were to choose the lowest value $x_{t}$ instead of reserve price $r_{t}$ which all buyers $v \geq x_{t}$ would submit in period $t$. Formally, the seller's decision rule is described as a function $\alpha_{t}$ from $\left(x_{0}, \cdots, x_{t-1}\right)$, the lowest values she chose up to period $t-1$, to $x_{t}$, where $x_{0}=v_{1}=1$. The function $\alpha_{t}$ satisfies in equilibrium.

$$
\begin{aligned}
\left(\beta_{t}\right)^{-1}\left(r_{t} \mid h_{t}\right) & =\left(\beta_{t}\right)^{-1}\left(s_{t}\left(h_{t-1}\right) \mid h_{t}\right) \\
& =x_{t}=\alpha_{t}\left(x_{0}, \cdots, x_{t-1}\right) .
\end{aligned}
$$

We can write cut-off value as $x_{t}=\alpha_{t}\left(v_{1}, \cdots, v_{t}\right)$ and $v_{t+1}=x_{t}$. In what follows, we consider $\left(\alpha_{t}, \beta_{t}\right)_{t=1,2, \cdots}$ instead of a PBE $(s, b, \mu)$. Consider buyer $v \geq\left(\beta_{t}\right)^{-1}\left(r_{t} \mid h_{t}\right)$ given $r_{t}$ and $h_{t}$. If the buyer $v$ submits a bid $v$ in period $t$, his expected payoff is given by:

$$
x G(x)-r_{t} G\left(r_{t}\right)-\int_{r_{t}}^{x} Y d G(Y)
$$

where $Y$ is the highest value of $n-1$ buyers and $G(x)=(F(x))^{n-1}$. Since his bid causes the other buyers' bids, he wins only if all the other buyers have reservation values below $v$. His payment is equal to reserve price $r_{t}$ if the second highest bid does not reach $r_{t}$, and otherwise he pays the second highest bid. Notice that the second highest bid is equal to the second highest value since all buyers use the symmetric strategy. On the other hand, if the buyer $v$ waits but expects to submit $v$ in the next period $t+1$, in the event of no sale, his expected payoff is given by:

$$
\delta\left[v G(v)-r_{t+1} G\left(r_{t+1}\right)-\int_{r_{t+1}}^{\left(\beta_{t}\right)^{-1}\left(r_{t} \mid h_{t}\right)} Y d G(Y)\right]
$$

Notice that $r_{t+1}=s_{t+1}\left(r_{1}, \cdots, r_{t}\right)=\beta_{t+1}\left(\alpha_{t}\left(v_{1}, \cdots, v_{t}\right), h_{t}\right)$. Given reserve price $r_{t}$ and history $h_{t}$, buyer $v$ such that $r_{t}=\beta_{t}\left(v, h_{t}\right)$ is indifferent between bidding in this period and bidding in the next period. Thus, by combining the equations above, we obtain an equation of $\alpha_{t}$ and $\beta_{t}$. Next we consider the seller's expected payoff. The seller's expected payoff satisfies the 
following equation.

$$
\begin{aligned}
\Pi\left(v_{1}, \cdots, v_{t}, x_{t}\right) & =n r_{t} G\left(r_{t}\right)\left[F\left(v_{t}\right)-F\left(\left(\beta_{t}\right)^{-1}\left(r_{t} \mid h_{t}\right)\right)\right]+ \\
& n \int_{\left(\beta_{t}\right)^{-1}\left(r_{t} \mid h_{t}\right)}^{v_{t}} \int_{r_{t}}^{\left(\beta_{t}\right)^{-1}\left(r_{t} \mid h_{t}\right)} Y d G(Y) d X+ \\
& n \int_{\left(\beta_{t}\right)^{-1}\left(r_{t} \mid h_{t}\right)}^{v_{t}} \int_{\left(\beta_{t}\right)^{-1}\left(r_{t} \mid h_{t}\right)}^{X} Y d G(Y) d X+\delta \Pi\left(v_{1}, \cdots, v_{t+1}, x_{t}\right) .
\end{aligned}
$$

That is, the seller's expected payoff has a recursive structure and she believes that she will face to buyers whose values are below $x_{t}$ in the next period $t+1$. She can sell her item for the price equal to the reserve price $r_{t}$ if only the highest buyer submits a bid in the first stage and no bid occurs in the second stage, and otherwise she obtains the second highest bid. The seller chooses $x_{t}$ in order to maximize her expected payoff given by the equation above. Their solution to the equations above give a $\operatorname{PBE}\left(\alpha_{t}^{*}, \beta_{t}^{*}\right)_{t=1,2, \cdots}$

\section{Stationary linear equilibrium}

As seen above, since the solution $\left(\alpha_{t}^{*}, \beta_{t}^{*}\right)_{t=1,2, \cdots}$ is very complex, we can hardly compare reserve prices in soft close auctions with hard close auctions. Thus, we specify the distribution and we consider a stationary linear PBE similar to McAfee and Vincent's (1997) linear example.

Definition 2 A PBE is stationary linear if the seller's and buyers' strategies are characterized by period independent two constants $(\alpha, \beta)$. The seller's strategy is given by $\alpha_{t}\left(v_{1}, \cdots, v_{t}\right)=$ $\alpha\left(v_{t}\right)=\alpha v_{t}$ for all $h_{t}$ and $t$, and buyers' strategies are given by $\beta_{t}\left(v, h_{t}\right)=\beta(v)=\beta v$ for all $h_{t}$ and $t$.

We assume that each buyer's valuation is uniformly distributed on the interval $[0,1] ; F(x)=$ $x$ and $G(x)=(F(x))^{n-1}$. Thus, the equilibrium reserve price in any period $t$ is given by $\alpha \beta v_{t}$. As noted by Cramton (1983), seller's on-path conjecture uniquely determines by Bayes' rule and we do not need to consider off-path conjecture, since the seller's belief can be calculated by Bayes' rule for all reserve prices.

Notice that we consider a linear PBE but we do not restrict each buyer's action to a linear action, so we allow them to deviate to any non-linear actions from a linear action. We show, 
however, that any best responses to linear strategies are also linear strategies.

Lemma 4 In any PBE, a best response to a linear strategy is a linear strategy.

For any reserve price $r$, if buyer $v$ submits $v$ in the first stage of the current period, conditional on winning, his expected payoff is given by:

$$
v G(v)-r G(r)-\int_{r}^{v} Y d G(Y)=\frac{v^{n}}{n}\left(1-\beta^{n}\right),
$$

where $Y$ is the highest of $n-1$ buyers' valuations. On the other hand, if he submits $v$ in the next period, his expected payoff is given by:

$$
\delta\left[v G(v)-\alpha r G(\alpha r)-\int_{\alpha r}^{\frac{r}{\beta}} Y d G(Y)\right]=\frac{\delta v^{n}}{n}\left[1-\alpha \beta^{n}\right] .
$$

Similar computations can be performed for $v \leq r / \beta$. Notice that given that the lowest type of buyer to submit a bid is strictly monotonic in the reserve price and bids are strictly monotonic in buyer type, in equilibrium, if $x$ is the lowest type to submit a bid with reserve price $r$, then $x$ will only win if no other buyer submits a bid, therefore if he wins he must win at exactly the reserve price.

For a buyer with value $v$ such that $r=\beta v$, these two payoffs are indifferent. By combining these equations, we obtain:

$$
\beta^{n}=\frac{1-\delta}{1-\delta \alpha^{n}} .
$$

Next, we consider the seller's expected payoff. Let $\Pi^{S}\left(v_{t}, x\right)$ be her expected payoff given that the seller believes that the support of the highest buyer's valuation and she sets a reserve price such that only buyers with valuations above $x$ submits bids in the first stage. The seller's expected payoff is given by:

$$
\Pi^{S}\left(v_{t}, x\right)=\underbrace{n r G(r)\left[F\left(v_{t}\right)-F\left(\frac{r}{\beta}\right)\right]}_{(1)}+\underbrace{n \int_{\frac{r}{\beta}}^{v_{t}} \int_{r}^{\frac{r}{\beta}} Y d G(Y) d X}_{(i i)}+\underbrace{n \int_{\frac{r}{\beta}}^{v_{t}} \int_{\frac{R}{\beta}}^{X} Y d G(Y) d X}_{(i i i)}+\underbrace{\delta \Pi^{S}\left(v_{t+1}, x\right)}_{(i v)} .
$$

The seller's expected payoff can be divided into four parts. (i) The first part is obtained when only one buyer submits a bid in the first stage and no buyer submits in the second stage. The 
price is given by the reserve price $r$. (ii) The second part is obtained when some buyers with $v \geq r / \beta$ submit bids in the first stage and some buyers with $v \in\left[r, \frac{r}{\beta}\right)$ submit counter bids. The price lies in an interval $\left(r, \frac{r}{\beta}\right)$. (iii) The third part is obtained when some buyers with $v \geq r / \beta$ submit bids in the first stage and no buyers submit in the second stage. The price lies in an interval $\left[\frac{r}{\beta}, v\right]$. (iv) The last part is a discounted continuation payoff. The distribution of the prices is described in the figure 1 .

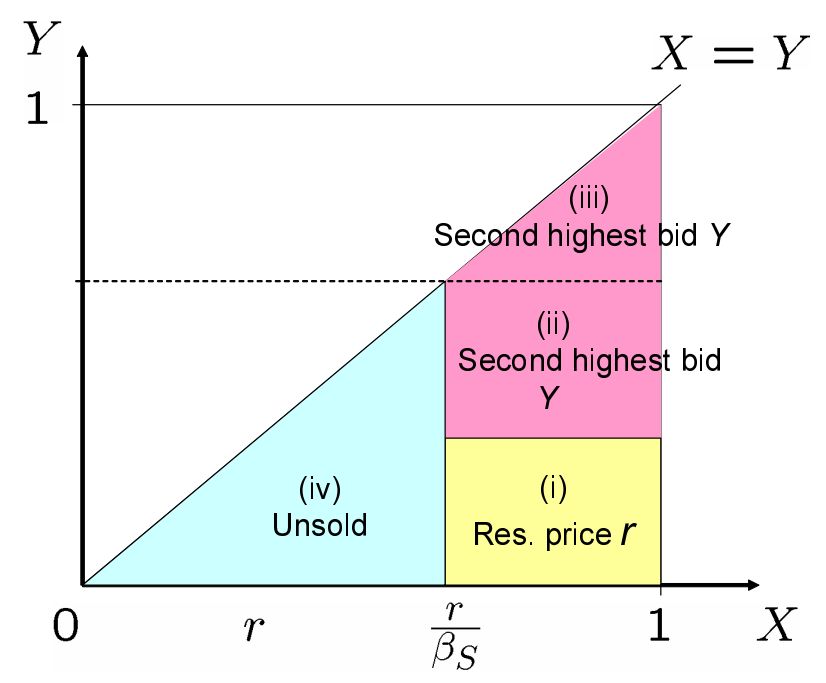

Figure 1: The distribution of the price

Consequently, we obtain the following proposition.

Proposition 1. There exists a unique stationary linear $\mathrm{PBE}\left(\alpha_{S}, \beta_{S}\right)$ such that

$$
\begin{aligned}
& \delta\left(\alpha_{S}\right)^{n+1}-2 \alpha_{S}+1=0 \\
& \left(\beta_{S}\right)^{n}=\frac{\alpha_{S}(n-1)(1-\delta)}{n\left(1-\alpha_{S}\right)-\alpha_{S}\left(1-\delta\left(\alpha_{S}\right)^{n}\right)} .
\end{aligned}
$$

In the equilibrium path, a reserve price in period $t$ is given by $\alpha_{S} \beta_{S} v_{t}$. The seller's belief is given by $v_{t}=\left(\alpha_{S}\right)^{t-1}$.

As can be seen in figure 2 , there exists the maximum value $\bar{\alpha}<1$, and the equilibrium 
value $\alpha(\delta)$ is in the open interval $(1 / 2, \bar{\alpha})$ depending on the discount factor $\delta \in(0,1)$ as shown in the following figure. We can obtain $\partial \alpha / \partial \delta>0$ and $\alpha \rightarrow \bar{\alpha}$ as $\delta \rightarrow 1$.

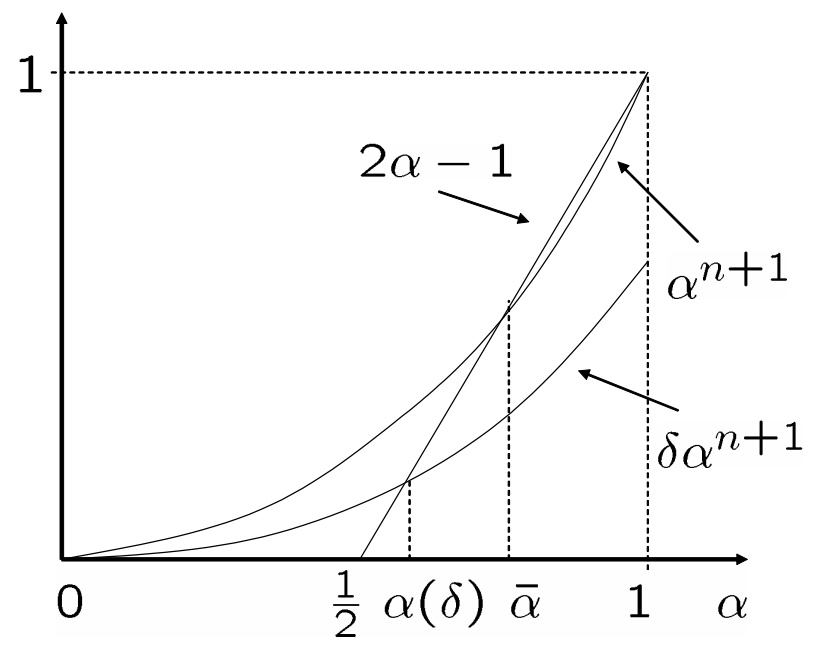

Figure 2: Optimal value of $\alpha$

Before proceeding the analysis, we briefly look at hard close auctions. Hard close auctions have only first stage, so each buyer cannot have any chances to reply other bids. McAfee and Vincent (1997) characterize a stationary linear PBE as follows:

Proposition 2 (McAfee and Vincent, 1997). In hard close auctions, there exists a unique stationary linear $\operatorname{PBE}\left(\alpha_{H}, \beta_{H}\right)$ such that

$$
\begin{aligned}
& \delta\left(\alpha_{H}\right)^{n+1}-2 \alpha_{H}+1=0 \\
& \beta_{H}=1+\frac{1}{n}\left[(1-\delta) \frac{\alpha_{H}}{1-\alpha_{H}}-1\right] .
\end{aligned}
$$

In the equilibrium path, a reserve price in period $t$ is given by $\alpha_{H} \beta_{H} v_{t}$. The seller's belief is given by $v_{t}=\left(\alpha_{H}\right)^{t-1}$. 


\section{Result}

In this section, we compare a PBE under a soft close and a PBE under a hard close. Let $\left(\alpha_{S}, \alpha_{S}\right)$ be a PBE under a soft close, and $\left(\alpha_{H}, \beta_{H}\right)$ be a PBE under a hard close. By Proposition 1 and Proposition 2, we immediately obtain $\alpha_{S}=\alpha_{H}$. This equation means that the seller decreases a reserve price at the same rate period by period independent of ending rules. In both auctions, the seller chooses a cut-off value $x_{t}$ in period $t$ such that all buyers with valuations $v \geq x_{t}$ submit bids. The cut-off value $x_{t}$ is given by $\alpha x_{t-1}$ for $t=1,2, \cdots$ and $x_{0}=1$. Since $\alpha_{S}=\alpha_{H}$, $\left(x_{S}\right)_{t}=\left(x_{H}\right)_{t}$ for all $t$. We summarize the result as the proposition below.

Proposition 3. Let $\left\{\left(x_{S}\right)_{t}\right\}_{t=1}^{\infty}$ be a sequence of cut-off values in soft close auctions and $\left\{\left(x_{H}\right)_{t}\right\}_{t=1}^{\infty}$ be a sequence of cut-off values in hard close auctions. In a stationary linear PBE $\left(x_{S}\right)_{t}=\left(x_{H}\right)_{t}$ holds for all $t$.

Though the cut-off value is given by $\alpha x_{t-1}$ in period $t$, the reserve price depends also on $\beta$ and it is given by $\alpha \beta x_{t-1}$ in period $t$. The following is the main result of this paper.

Theorem 1. Let $\left\{\left(r_{S}\right)_{t}\right\}_{t=1}^{\infty}$ be a sequence of reserve prices in soft close auctions and $\left\{\left(r_{H}\right)_{t}\right\}_{t=1}^{\infty}$ be a sequence of reserve prices in hard close auctions. In a stationary linear PBE $\left(r_{S}\right)_{t}<\left(r_{H}\right)_{t}$ holds for all $t$.

The intuition of Theorem 1 is following. In soft close auctions, since each buyer has a chance to react to the other buyers' bids in the second stage, the buyer has an incentive to wait to submit a bid until a reserve price will go down. However, the seller knows the buyers' incentives for waiting, thus she rationally charge a lower reserve price in order to prevent her payoff from discounting in soft close auctions than in hard close auctions. Figure 3 shows a numerical example where $\delta=0.99$ and $n=10$. In such a case, we obtain $\alpha=0.500243$, $\beta_{S}=0.631019$ and $\beta_{H}=0.9010097$. 


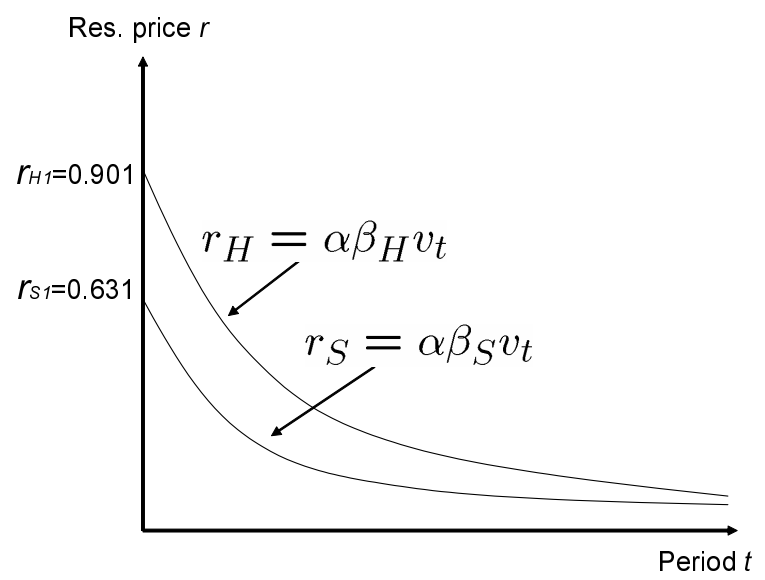

Figure 3: Reserve price (numerical example)

Corollary 1. From the view point of the seller, revenue equivalent holds. For all $t$,

$$
\Pi^{S}\left(v_{t}, x_{t}^{S}\right)=\Pi^{H}\left(v_{t}, x_{t}^{H}\right)
$$

Notice that Corollary 1 crucially depends on the private value setting, but may not depend on the uniform distribution.

\section{Empirical Data}

\subsection{Data description}

In this section we analyze reserve price data of Yahoo! Japan auction. In Yahoo! Japan auction, a seller chooses the ending rule from a soft close and a hard close. Though a default option is a hard close, a seller can freely choose a soft close. Data was downloaded from each auction in a category "System" in "Nintendo DS" in "Toys". The data is publicly obtained and freely downloaded during the auctions. The data set consists of auctions except auction stores between July 2008 and August 2008. We focus on reserve prices, all items should be similar enough, so auctions which treat the following items were excluded. (i) New items; a stable re-sale market exists in which the items can be sold for nearly list prices. (ii) Broken items; these items are considered as the other items. The quality among items should be similar. (iii) Item bundle; items with some softs or souvenir are also considered as the different 
items. Yahoo! Japan auction equips with many options for a seller "Take-it-price" auctions and "Buy-it-now" auctions in which a bid equal to a seller-defined take-it-price immediately halts the auction is accepted, and "Secret reserve price" auctions were also excluded. In the former auctions, even though a hammer price may not reach the take-it-price, the item will be sold to a winner, but in the latter auctions a starting price is posted as the buy-it-now price. 14 In total, the data set consists of 264 auctions, 211 for soft close auctions and 53 for hard close auctions. For each auction, I recorded a reserve price (starting price). I also collected information about each seller's feedback number. On Yahoo! Japan auction, buyers and sellers have the opportunity to give each other a positive feedback $(+1)$, a neutral feedback $(0)$ or a negative feedback (-1). ${ }^{15}$ The cumulative total of positive and negative feedback is what we call the "feedback number" on Yahoo! Japan.

\subsection{Average reserve price}

Table 1 and Graph 1 show a distribution of reserve prices. In soft close auctions one third of reserve prices (73 prices) are 1 yen (minimum price) or less than 2000 yen while only $11 \%$ of reserve prices (6 prices) are set under 2000 yen in hard close auctions. The maximum reserve price in soft close auctions is 16800 yen, which is the same as the list price, while the maximum reserve price in hard close auctions is 13500 yen. The auctions with reserve prices over 10001 yen are $37(17.5 \%)$ in soft close auctions and $14(26.4 \%)$ in hard close auctions.

Table 2 shows a comparison between the average reserve price of soft close auctions and hard close auctions. As can be seen in Table 2, sellers post higher reserve prices in hard close auctions than in soft close auctions, and the difference is statistically significant (two-sided Student's $t$-test, $p=0.013)$. The result confirms the theoretical result that sellers post higher reserve prices in hard close auctions than in soft close auctions. ${ }^{16}$

\footnotetext{
${ }^{14}$ Roth and Ockenfels (2002) and Ockenfels and Roth (2006) selected the category Computers to analyze IPV auctions. Though the category Computers consists of a variety of items, there is no matter since Roth and Ockenfels (2002) and Ockenfels and Roth (2006) focus on bidding time but the retail price. However, since we want to focus on the reserve price, items should be similar enough. Therefore, I selected the category "System" in "Nintendo DS".

${ }^{15}$ The feedback system on Yahoo! Japan is similar to eBay, but the positive feedback includes "Very good" and "Good", and the negative feedback includes "Very bad" and "Bad".

${ }^{16}$ We do not reject the null-hypothesis that the variance of reserve prices in soft close auctions is the same as the variance of reserve prices in hard close auctions (one-sided $F$-text, $p=0.071$ ).
} 
Table 1: Distribution of reserve prices of soft close auctions and hard close auctions on Yahoo! Japan

\begin{tabular}{lrr}
\hline JPY & Soft close & Hard close \\
\hline $1-2000$ & 73 & 6 \\
$2001-4000$ & 8 & 2 \\
$4001-6000$ & 26 & 12 \\
$6001-8000$ & 24 & 8 \\
$8001-10000$ & 43 & 11 \\
$10001-$ & 37 & 14 \\
\hline Observation & 211 & 53 \\
\hline
\end{tabular}

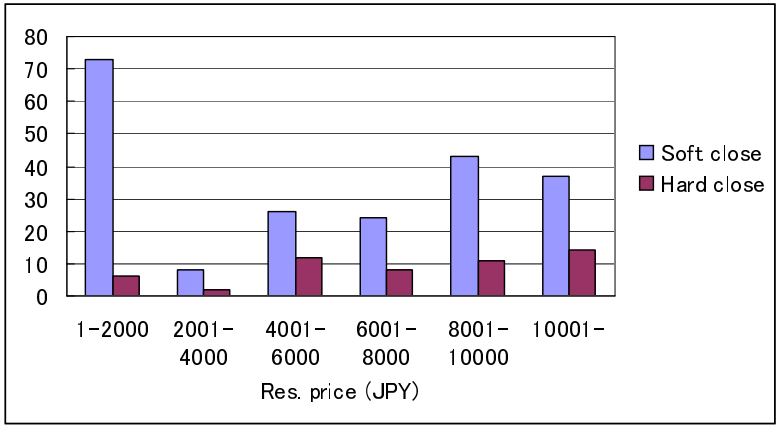

Graph 1: Distribution of reserve prices

Table 2: Average reserve prices of soft close auctions and hard close auctions on Yahoo! Japan

\begin{tabular}{lrr}
\hline & Soft close (JPY) & Hard close (JPY) \\
\hline Mean & 5962.5 & 7682.7 \\
Variance & 21741389.9 & 15414840.4 \\
Observation & 211 & 53 \\
\hline
\end{tabular}


Table 3: Variables

\begin{tabular}{lr}
\hline RESPRICE & Reserve prices (JPY) \\
ENDRULE & Hard close (1) or Soft close (0) \\
RATING & Seller's rating number (positive or negative integer) \\
\hline
\end{tabular}

\subsection{Regression analysis}

The following regression analysis shed light on how ending rules relate to reserve prices. We have one dependent variable RESPRICE and two explanatory variables ENDRULE and RATING in the regression analysis in Table 3. ENDRULE is a binary variable with value 1 if the seller chooses a hard close and 0 otherwise (a soft close). RATING is the seller's rating or feedback number. Feedback is made up of comments and ratings left by the seller (buyer) whom you have bought from (sold to). These comments and ratings are considered as valuable indicators of the reputation as a buyer or seller on Yahoo! Japan. The regression confirm the theoretical prediction that ending rules significantly affect the reserve prices and reserve prices in hard close auctions are higher than in soft close auctions. The regression equation is given by:

$$
R E S P R I C E=\alpha+\beta_{1} R A T I N G+\beta_{2} E N D R U L E
$$

The result of the regression is given by:

$$
R E S P R I C E=5768.3+0.295 R A T I N G+1812.7 E N D R U L E
$$

\section{Conclusion}

We considered how rules for ending auctions affect the reserve prices and the buyers' bidding behaviors. In Internet auctions, items being unsold will be re-auctioned since the cost is very low. Since the reserve price decreases and buyers correctly anticipate the decrease of reserve prices, they have incentives to wait for the next period. Since buyers faced to a hard close are more impatient than buyers faced to a soft close, the former submit bids against a high 
Table 4: Regression result

\begin{tabular}{lrr}
\hline & Coefficient & Standard Error \\
\hline Intercept $(\alpha)$ & 5768.3 & 352.3 \\
& $(0.000)$ & \\
RATING & 0.295 & 0.264 \\
& $(0.265)$ & \\
ENDRULE & 1812.7 & 699.0 \\
& $(0.010)$ & \\
\hline$R^{2}$ & 0.028 & \\
Adjusted $R^{2}$ & 0.020 & \\
SE & 4524.0 & \\
\hline
\end{tabular}

reserve price. A seller in auctions with a hard close chooses higher reserve prices than a seller in auctions with a soft close. Furthermore, we can investigate the above results by using empirical data. For example, the seller can choose rules for ending her auction in Yahoo! Japan. There are many categories in this auction. According to Ockenfels and Roth (2006), "information about the retail price of most items is in general easily available since most items are new". The category of DS in Yahoo! Japan may seem to be suitable. This is the future research.

\section{References}

1. Ashenfelter, O. (1989), "How Auctions Work for Wine and Art," Journal of Economic Perspectives 3, 23-36

2. Cramton, P. C. (1984), "Bargaining with Incomplete Information: An Infinite Horizon Model with Continuous Uncertainty", Review of Economic Studies 51, 579-594

3. Fudenberg, D., Levine, D. and Tirole, J. (1985), "Infinite-Horizon Models of Bargaining with One-Sided Incomplete Information", in A. Roth (ed.), Game Theoretic Models of Bargaining, Cambridge: Cambridge University Press.

4. Grant, S., Kajii, A., Menezes, F. and Ryan, M. J. (2006), "Auctions with Options to Re-auction", International Journal of Economic Theory 2, 17-39

5. Gul, F., Sonnecschein, H. and Wilson, R. (1986), "Foundations of Dynamic Monopoly 
and the Coase Conjecture", Journal of Economic Theory 39, 155-190

6. Krishna, V. (2002), Auction Theory, Elservier: Academic Press.

7. Lucking-Reiley, D. (2000), "Auctions on the Internet: What's being Auctioned, and How?", The Journal of Industrial Economics 48, No.3, 227-252

8. McAfee, R. P., and D. Vincent (1997), "Sequentially Optimal Auctions", Games and Economic Behavior 18, 246-276

9. Milgrom, P. R., and R. J. Weber (1982), "A Theory of Auctions and Competitive Bidding, II", in P. Klemperer (ed.), The Economic Theory of Auctions, Cheltenham, U.K.: Edward Elgar.

10. Ockenfels, A. and Roth, A. E. (2006), "Late and multiple bidding in second price Internet auctions: Theory and evidence concerning different rules for ending an auction", Games and Economic behavior 55, 297-320

11. Roth, A. E., and Ockenfels, A. (2002), "Last-minute Bidding and The Rules for Ending Second-price Auctions: Evidence from eBay and Amazon Auctions on the Internet", American Economic Review 92:1093-1103

12. Samuelson, W. (1984), "Bargaining under Asymmetric Information", Econometrica 52, 992-1005

13. Sobel, J. and Takahashi, I. (1983), "A Multistage Model of Bargaining", Review of Economic Studies, 50:411-426

14. Weber, R. (1983), "Multiple Object Auctions", in R. Engelbrecht-Wiggans, M. Shubik, and R. Stark (eds.), Auctions, Bidding and Contracting: Uses and Theory, New York, NY.: New York University Press, 165-191

\section{Appendix}

\section{Proof of Lemma 1}


Proof. In the second stage, since the auction surely ends, the auction is the same as a standard sealed-bid-second-price auction, so submitting a true value is a weakly dominant strategy for buyer $v$. Conditioning on bidding, the buyer $v$ should submit $v^{\prime} \leq v$ in the first stage. Otherwise, he cannot submit $v$ in the second stage by assumption.

\section{Proof of Lemma 2}

Proof. Following history $h_{t}$, we consider a case where buyer $v$ submits a bid $v$ in period $t$. Since the other buyers submit true values in the second stage after buyer $v$ submits in the first stage, we obtain the following inequality in equilibrium:

$$
v G(v)-R_{t} G\left(R_{t}\right)-\int_{R_{t}}^{v} Y d G(Y) \geq \delta V\left(v, h_{t}\right) \operatorname{Prob}[\text { No bid }]
$$

where $Y$ is the highest value of the other $n-1$ buyers and $V\left(v, h_{t}\right)$ is a continuation payoff for buyer $v$. Suppose, contrarily, that buyer $v^{\prime}(>v)$ does not submit a bid in equilibrium. That is, the following inequality must hold:

$$
v^{\prime} G\left(v^{\prime}\right)-R_{t} G\left(R_{t}\right)-\int_{R_{t}}^{v^{\prime}} Y d G(Y) \leq \delta V\left(v^{\prime}, h_{t}\right) \operatorname{Prob}[\text { No bid] } .
$$

Therefore, we obtain:

$$
\delta\left(V\left(v^{\prime}, h_{t}\right)-V\left(v, h_{t}\right)\right) \frac{\operatorname{Prob}[\mathrm{No} \text { bid }]}{G(v)}>v^{\prime}-v
$$

In equilibrium, buyer $v$ cannot benefit from mimicking buyer $v^{\prime}$,

$$
\begin{aligned}
V_{B}\left(v, h_{t}\right) & =\sum_{\tau=t}^{\infty} \delta^{\tau} \rho_{\tau}\left(v, h_{t}\right)\left(v-p_{\tau}\left(v, h_{t}\right)\right) \\
& \geq \sum_{\tau=t}^{\infty} \delta^{\tau} \rho_{\tau}\left(v^{\prime}, h_{t}\right)\left(v-p_{\tau}\left(v^{\prime}, h_{t}\right)\right) \\
V_{B}\left(v^{\prime}, h_{t}\right) & =\sum_{\tau=t}^{\infty} \delta^{\tau} \rho_{\tau}\left(v^{\prime}, h_{t}\right)\left(v^{\prime}-p_{\tau}\left(v^{\prime}, h_{t}\right)\right)
\end{aligned}
$$

Finally, we obtain:

$$
\delta \sum_{\tau=t}^{\infty} \delta^{\tau} \rho_{\tau}\left(v^{\prime}, h_{t}\right)\left(v^{\prime}-v\right) \frac{\operatorname{Prob}[\text { No bid }]}{G(v)}>v^{\prime}-v
$$




\section{Proof of Lemma 3}

Proof. Fix a history $h_{t-1}$. Let $\beta_{t}\left(v, h_{t}\right)$ be a cut-off value chosen by buyer $v$ given $h_{t-1}$ in period $t$ in equilibrium. Since buyers use the cut-off strategy defined by equation $(1), \beta_{t}\left(v, h_{t}\right)$ is increasing in $v$ given $h_{t}$ by Lemma 2. Thus, for any reserve price $r_{t}$, there exists a unique value $v$ such that $r_{t}=\beta_{t}\left(v, h_{t}\right)$. Notice that there would be a case $v>1$. Suppose that the seller chooses reserve prices $\left.r_{1}, \cdots, r_{t}\right)$ up to period $t$, and no bid is submitted in period $t$. Since there should exist the lowest value $x_{t}$ such that $r_{t}=\beta_{t}\left(x_{t}, h_{t}\right)$ where $h_{t}=\left(r_{1}, \cdots, r_{t-1}\right)$ in period $t$, the seller will believe that the highest value for the buyer she faces to is below $v_{t+1}=x_{t}$ in the next period $t+1$. Therefore, the support of $\mu\left(h_{t}\right)$ is given by $\left[0, v_{t+1}\right]$ in period $t+1$ in equilibrium. Furthermore, since the seller has the initial belief at the beginning of the game, so $v_{1}=1$.

\section{Proof of Lemma 4}

Proof. Let be $r^{*}$ an equilibrium strategy. For any $r$ and $v$, the following inequality holds.

$$
\sum_{t=1}^{\infty} \delta^{t-1}\left[v-\alpha_{t}\left(r^{*}, v\right) p_{t}\left(r^{*}, v\right)\right] \geq \sum_{t=1}^{\infty} \delta^{t-1}\left[v-\alpha_{t}(r, v) p_{t}(r, v)\right]
$$

where $\alpha_{t}(r, v)$ is an expected probability of obtaining the object and $p_{t}(r, v)$ is an expected hammer price in period $t$ given $r$ and $v p_{t}(r, v)$. If at least one bid is submitted, the second stage arises, so $p_{t}\left(r^{*}, v\right)=p_{t}(r, v)$ for any deviation $r$ in period $t . \alpha_{t}(r, v)=1$ in a period where the buyer submits a serious bid, and $\alpha_{t}(r, v)=0$ in other periods. Therefore, the buyer's strategy decides the period to submit given a sequence of reserve prices. If the buyer cannot benefit from any linear strategies $r$, then he cannot benefit from any (non-linear) strategies.

\section{Proof of Proposition 1}


Proof. The seller's expected payoff is given by:

$$
\begin{aligned}
\Pi^{S}\left(v_{t}, x\right)= & n r G(r)\left[F\left(v_{t}\right)-F\left(\frac{r}{\beta}\right)\right]+n \int_{\frac{r}{\beta}}^{v_{t}} \int_{r}^{\frac{r}{\beta}} Y d G(Y) d X \\
& +n \int_{\frac{r}{\beta}}^{v_{t}} \int_{\frac{r}{\beta}}^{X} Y d G(Y) d X+\delta \Pi^{S}\left(v_{t+1}, x\right) \\
= & n(\alpha x)^{n}\left(v_{t}-x\right)+(n-1)\left(v_{t}-x\right)\left(1-\alpha^{n}\right) x^{n} \\
& +n(n-1)\left[\frac{v_{t}}{n}\left(\left(v_{t}\right)^{n}-x^{n}\right)-\frac{1}{n+1}\left(\left(v_{t}\right)^{n+1}-x^{n+1}\right)\right]+\delta \Pi^{S}\left(v_{t+1}, x\right) .
\end{aligned}
$$

The last part $\Pi^{S}\left(v_{t+1}, x\right)$ is a continuation payoff at period $t$. We solve the following maximization problem with respect to $x$.

$$
\max _{x} \Pi^{S}\left(v_{t}, x\right)
$$

Let $x^{*}\left(v_{t}\right)$ be a solution to the above problem. The continuation payoff can be written as:

$$
\Pi^{S}\left(v_{t+1}, x^{*}\left(v_{t}\right)\right)=\Pi^{S}\left(x^{*}\left(v_{t}\right), x^{*}\left(v_{t}\right)\right)=\tilde{\Pi}^{S}\left(x^{*}\left(v_{t}\right)\right)
$$

At period $t-1, v_{t}=x^{*}\left(v_{t-1}\right)$ is selected to maximize the seller's expected payoff, therefore we obtain:

$$
\tilde{\Pi}^{S}\left(v_{t}\right)=\Pi^{S}\left(v_{t}, x^{*}\left(v_{t}\right)\right)
$$

To solve the maximization problem, we first obtain the derivative of the continuation payoff $\tilde{\Pi}^{S}\left(v_{t}\right)$. By using Envelop Theorem, the derivative of the continuation payoff is given by:

$$
\begin{aligned}
\frac{\partial \tilde{\Pi}^{S}\left(v_{t+1}, v_{t}\right)}{\partial v_{t}} & =\frac{d \Pi^{S}\left(v_{t}, x^{*}\left(v_{t}\right)\right)}{d v_{t}}=\left.\frac{\partial \Pi^{S}\left(v_{t}, x\right)}{\partial v_{t}}\right|_{x=x^{*}\left(v_{t}\right)} \\
& =n\left(\alpha x^{*}\right)^{n}+(n-1)\left(1-\alpha^{n}\right)\left(x^{*}\right)^{n}+n(n-1)\left[\frac{1}{n}\left(v_{t}\right)^{n}-\left(x^{*}\right)^{n}+v_{t}^{n}-v_{t}^{n}\right] \\
& =n\left(\alpha x^{*}\right)^{n}-n\left(\alpha x^{*}\right)^{n}+n\left(x^{*}\right)^{n}-\left(1-\alpha^{n}\right)\left(x^{*}\right)^{n}+(n-1)\left(v_{t}^{n}-\left(x^{*}\right)^{n}\right) \\
& =\left[(\alpha \beta)^{n}+n-1\right] v_{t}^{n}
\end{aligned}
$$

In the above computation, we use $x^{*}=x^{*}\left(v_{t}\right)=\alpha v_{t}$. Finally, we consider the maximization problem of $\Pi^{S}\left(v_{t}, x\right)$ w.r.t $x$. The first order condition is given by:

$$
\begin{aligned}
\frac{\partial \Pi^{S}\left(v_{t}, x\right)}{\partial x}=0 & =n^{2} \alpha^{n} x^{n-1}\left(v_{t}-x\right)-n(\alpha x)^{n} v_{t}+(n-1)\left(1-\alpha^{n}\right)\left[n\left(v_{t}-x\right) x^{n-1}-x^{n}\right] \\
& +n(n-1)\left(-v_{t} x^{n-1}+x^{n}\right)+\delta x^{n}\left[(\alpha \beta)^{n}+n-1\right]
\end{aligned}
$$


Therefore, we obtain the following equations:

$$
\begin{aligned}
& \delta\left(\alpha_{S}\right)^{n+1}-2 \alpha_{S}+1=0 \\
& \left(\beta_{S}\right)^{n}=\frac{\alpha_{S}(n-1)(1-\delta)}{n\left(1-\alpha_{S}\right)-\alpha_{S}\left(1-\delta\left(\alpha_{S}\right)^{n}\right)} .
\end{aligned}
$$

\section{Proof of Proposition 2.}

Proof. Similar to soft close, we can consider the buyer's expected payoff. The buyer with valuation $x=r / \beta$ is indifferent between bidding in the current period and bidding in the next period, therefore we obtain the following equation.

$$
x G(x)-r G\left(\frac{r}{\beta}\right)-\int_{\frac{r}{\beta}}^{x} Y d G(Y)=\delta\left[x G\left(\frac{r}{\beta}\right)-\alpha r G\left(\frac{\alpha r}{\beta}\right)-\int_{\frac{\alpha r}{\beta}}^{\frac{r}{\beta}} Y d G(Y)\right] .
$$

On the other hand, the seller's expected payoff is given by:

$$
\Pi^{H}\left(x, v_{t}\right)=n \cdot r(x) G(x)\left[F\left(v_{t}\right)-F(x)\right]+n \int_{x}^{v_{t}} \int_{x}^{X} Y d G(Y) d X+\delta \tilde{\Pi}^{H}(x) .
$$

These equations give the constants $\left(\alpha_{H}, \beta_{H}\right)$ such that:

$$
\begin{aligned}
& \delta\left(\alpha_{H}\right)^{n+1}-2 \alpha_{H}+1=0 \\
& \beta_{H}=1+\frac{1}{n}\left[(1-\delta) \frac{\alpha_{H}}{1-\alpha_{H}}-1\right] .
\end{aligned}
$$

\section{Proof of Theorem 1.}

Proof. In any period $t$, the reserve price is given by $r_{S t}=\alpha_{S} \beta_{S} v_{t}$ and $r_{H t}=\alpha_{H} \beta_{H} v_{t}$, respectively. Since $\alpha_{S}=\alpha_{H}=\alpha$, it is sufficient to show that $\beta_{S}<\beta_{H}$.

By Proposition 1 and Proposition 2,

$$
\begin{aligned}
\left(\beta_{S}\right)^{n} & =\frac{1-\delta}{1-\delta \alpha^{n}}=1-\frac{1-2 \alpha+\delta \alpha}{1-\alpha} \\
\beta_{H} & =1-\frac{\delta}{n} \cdot \frac{1-\alpha^{n}}{1-\delta \alpha^{n}}=1-\frac{1-2 \alpha+\delta \alpha}{n(1-\alpha)} .
\end{aligned}
$$


Let $z=(1-2 \alpha+\delta \alpha) /(1-\alpha)$, therefore we obtain

$$
\beta_{H}=1-\frac{z}{n} \text { and }\left(\beta_{S}\right)^{n}=1-z .
$$

Let $w(z)=\left(1-\frac{z}{n}\right)^{n}-(1-z)$. We can show that $w(z)>0$ for all $z$ by Lemma 5 , and we complete the proof.

\section{Proof of Lemma 5.}

Lemma 5. $w(z)>0$ for all $z$.

Proof.

$$
\begin{aligned}
z & =\frac{1-s \alpha+\delta \alpha}{1-\alpha}=\frac{1-2 \alpha+\frac{2 \alpha-1}{\alpha^{n}}}{1-\alpha}=\frac{\left(\alpha^{n}-1\right)(1-2 \alpha)}{\alpha^{n}(1-\alpha)} \\
& =\frac{2 \alpha-1}{\alpha^{n}} \times\left(1+\alpha+\cdots \alpha^{n-1}\right)=\frac{2 \alpha-1}{\alpha^{n+1}} \times\left(\alpha+\alpha^{2}+\cdots \alpha^{n}\right) \\
& <\alpha+\alpha^{2}+\cdots \alpha^{n}<n .
\end{aligned}
$$

The above inequality holds from the fact $\alpha^{n+1}>2 \alpha-1$. Thus, $z<n$. Finally, $w(0)=0$ and

$$
w^{\prime}(z)=-\left(1-\frac{z}{n}\right)^{n-1}+1>0 .
$$

\section{Proof of Corollary 1.}

Proof. Since the last two parts of the seller's expected payoff under the both ending rules are the same, it is sufficient to compare the first part of the seller's expected payoff under a hard close as the sum of the first and the second part of expected payoff under a soft close. By using the fact $\alpha^{S}=\alpha^{H}=\alpha$, we obtain the following equation under a hart close:

$$
\begin{aligned}
{[\text { Hard }] } & =n \beta^{H} x^{n} \\
& =n x^{n}\left[1-\frac{\delta}{n} \cdot \frac{1-\alpha^{n}}{1-\delta \alpha^{n}}\right]
\end{aligned}
$$

We obtain the following equation under a soft close:

$$
\begin{aligned}
{[\text { Soft }] } & =n x^{n} \cdot \frac{1-\delta}{1-\delta \alpha^{n}}+(n-1) x^{n} \cdot \frac{\delta\left(1-\alpha^{n}\right)}{1-\delta \alpha^{n}} \\
& =\frac{x^{n}}{1-\delta \alpha^{n}}\left[n\left(1-\delta \alpha^{n}\right)-\delta\left(1-\alpha^{n}\right)\right]
\end{aligned}
$$

Therefore, we obtain $[$ Hard $]=[$ Soft $]$. 